\title{
Processing and Prospect of Electronic Nose
}

\author{
Yibo Deng $^{1}$ \\ ${ }^{1}$ College of Communication Engineering, Xidian University, Xi'an, Shaanxi, 710126, China
}

\begin{abstract}
Recently, a Novel Coronavirus Pneumonia (NCP) swept the globe. This kind of new virus has extremely high infection efficiency, which has brought great disaster to human's production and daily life. However, it is reported that lung cancer and other diseases with a high incidence will lead to a very high mortality rate if they are not diagnosed and treated in time. Therefore, it is urgent to design an accurate and convenient new diagnostic equipment. Due to the increasing innovation of sensor module in the field of Information and Communication Technology (ICT), electronic nose emerges as the times require. The device can use sensors to analyze Volatile Organic Compounds exhaled by people with certain diseases and identify chemical components of various odor and flavor. In addition, many other types of sensors and classification methods such as machine learning (ML) algorithms are also mentioned in this paper. At the end of the paper, the author attached a related experiment and some shortcomings and prospects of electronic nose as well.
\end{abstract}

\section{Introduction}

Lung cancer has become one of the most commonly types of cancer related to deaths. The number of lung cancer related deaths even exceed the sum of breast, colon, liver and prostate cancer [1]. The mortality rate of patients could be extremely high if they are not diagnosed in early stage within five years. The survival percentage could drop to less than $5 \%$ because of the spread of their tumors. But fortunately, if the lung cancer in the early stage can be found as soon as possible, the death rate of patients can jump sharply to more than $70 \%$ [2]. Therefore, it is urgent to develop a new accurate and convenient diagnostic method.

In fact, using low dose chest Computed Tomography (CT) scan, which is particularly effective in the diagnosis of some small size lung cancer, has been a successful technique. Nevertheless, the result of it could easily include high rate of false positive. In addition, the radiation of CT scan can induce cancers [2]. Therefore, breath analysis has gradually attracted scientists' attention.

By the analysis of human respiratory components, the comprehensive information of human metabolism can be enhanced. It indicated that there are more than 200 kinds of Volatile Organic Compounds (VOCs) in human breath, which are transported by human blood to the lungs, then removed through respiration. Thus, it is reported that the abnormality of some VOCs in human body may indicate the occurrence of some diseases. For instance, the abnormal values of mercaptans and alkanes could be closely related with liver disorders and the value of exhaled methyl nitrate is one of the noninvasive markers of diabetes [3].
Even though the accuracy of the methods above is relatively high when identifying VOCs in breath, there are still many shortcomings which are difficult to solve out. These methods require many expensive, complex and non-portable instruments with large scale, and respiratory samples need to be concentrated before entering these devices for analysis [4]. These disadvantages make it difficult for them to be widely used in the medical field. Hence, developing a new type of electronic nose is the target of many scientists. The electronic noses will sense and recognize the target gas with high precision, when the appropriate sensor is being used. Differing from those methods above, the electronic nose could be very cheap and easy to set up and use.

Because of its excellent performance, electronic nose has been widely used in various fields, such as food, agriculture, transportation, military and so on. This paper aims to summarize the previous work and contribute to the research and development of new electronic nose in the future.

\section{Related works}

There are three main part in e-noses: sensor unit, electronic unit and pattern recognition unit. The electrical signal generated by the sensor unit depends on the characteristics of the chemical composition applied to the electronic nose. After the sensor senses the odour, the capacitance, conductivity and frequency of the sensor unit will change accordingly, and then the generated signal will be transmitted to the computer for analysis. According to different target gases, the most sensitive sensor can be chosen to achieve the best effect [3] Transformers, oscillator circuits and filters make up the 
electronic unit, which is a part of obtaining the electrical signals properly. Pattern recognition is the last unit. And attributes determined by electronic signals are used to define as the recognition and classification of odours.

Electronic nose is a combination of such a series of sensors and pattern recognition system. [5], the researchers collected the data of exhaled breath of smokers and non-smokers, then detected, extracted and classified the odour, and finally imported it into the computer to predict their cancer risk.
Support vector machine (SVM) refers to the support vector machine, which is a common classification method. In the field of machine learning, it is a supervised learning model, which is usually used for pattern recognition, classification and regression analysis.

\subsection{Previously proposed design of E-nose}

Figure 1 below shows one type of electronic nose block diagram proposed by previous scientists.

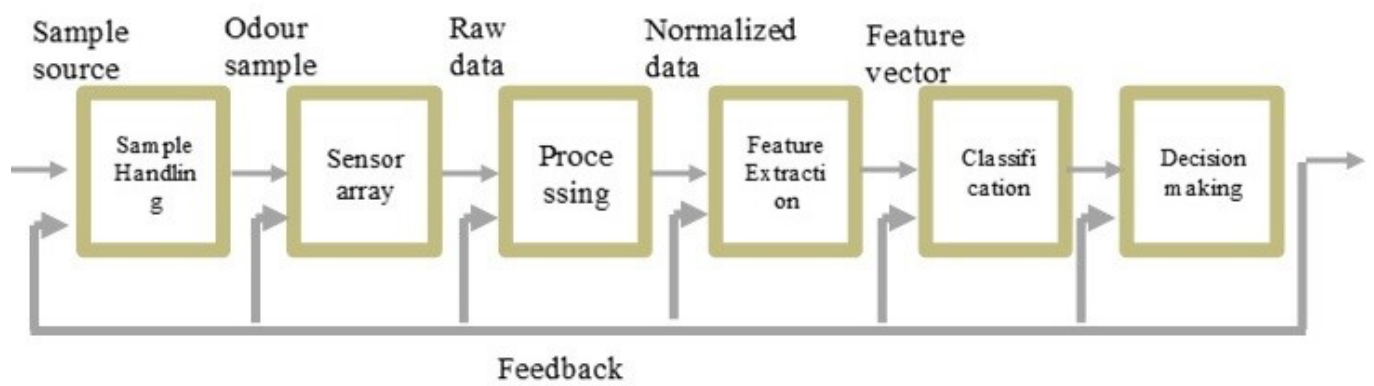

Fig. 1 Proposed E-nose block diagram

Firstly, the collected odour samples should be filtered and then transported to the sensor array to convert the chemical signals into corresponding electronic signals. Then the signal is imported into the computer for noise removal and other pre-processing. Then the cancer was classified according to fatigue, weight change and other characteristics [6]. In this process, the reasonable use of Machine Learning (ML) algorithm can help more accurately predict lung cancer.
At the same time, in order to obtain better accuracy, multiclass SVM is employed [7]. SVM, which is a supervised ML algorithm essentially, is used to construct a group of hyperplanes in infinite dimensional space.

The commonly used sensors of electronic nose are Mental Oxide Field Effect Transistor (MOSFET) sensor, Surface Acoustic Wave (SAW) sensor, conducting gas sensor and optical sensor.

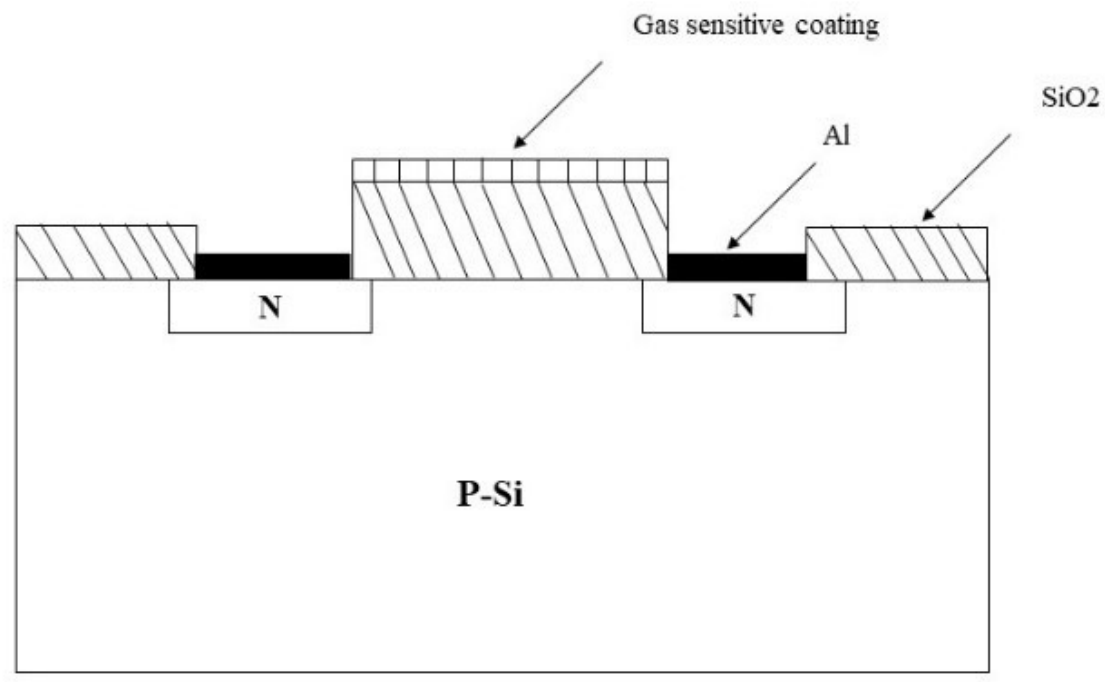

MOS Sensor

Fig. 2 MOS sensor

The MOSFET is a kind of transducer, which could be used to control voltage and transform chemical changes into electrical signal [8]. When voltage is applied across the gate and drain terminal, the element will generate an electric field, which is recorded by the transistor. It should be noted that when the grid layer is thick, the sensitivity of the sensor to non-hydrogen molecules in the gas will be reduced. Therefore, a thinner gate layer is necessary in improving the precision of the sensor. 


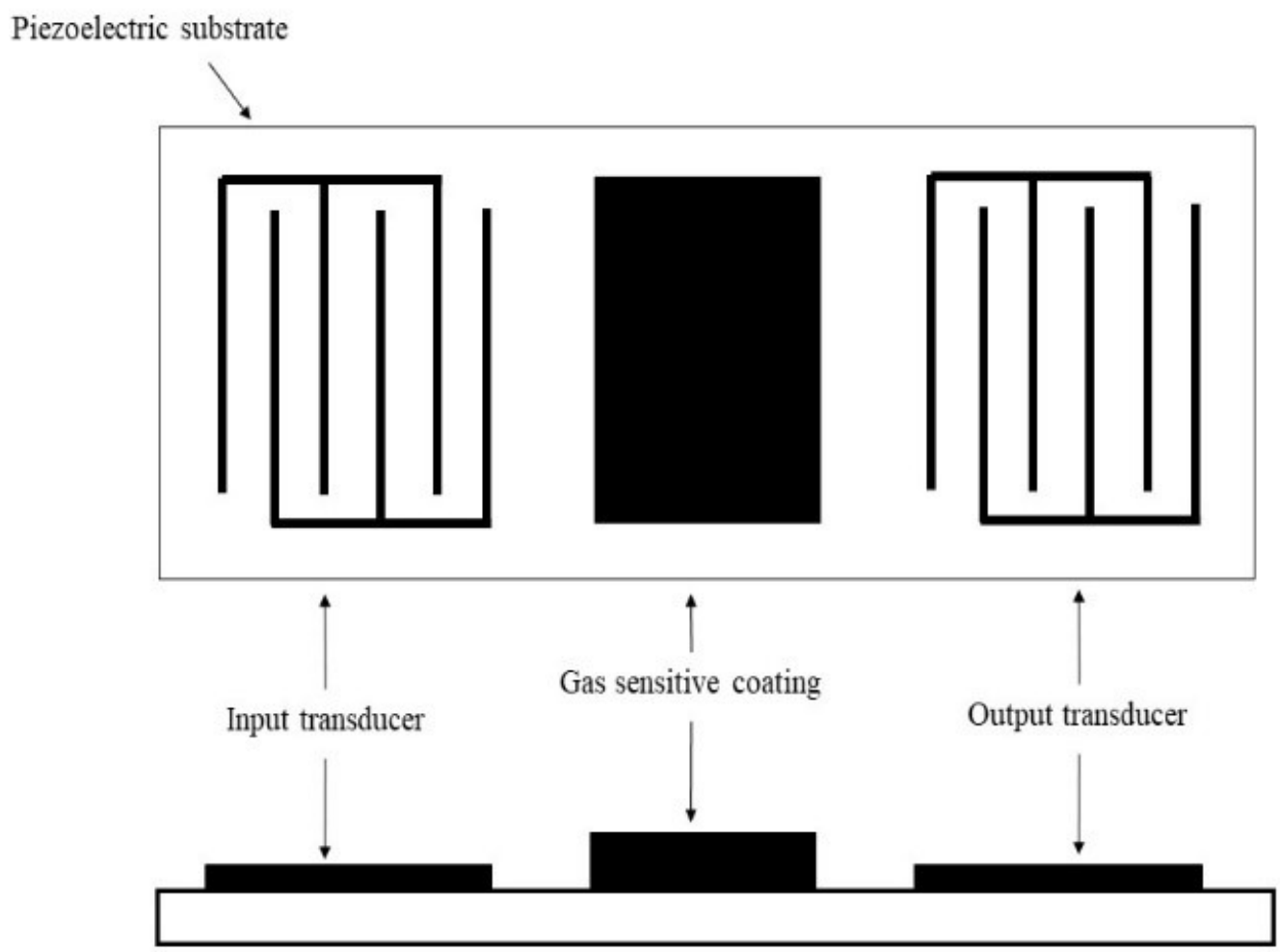

Fig.3 SAW sensor

SAW sensor is a strain sensor based on surface acoustic wave technology. It is widely used because of its advantages of high precision, high sensitivity, small size, light weight, low power consumption and so on [9]. It is easy to transmit and process digital frequency signal with SAW sensor as well. As shown in Figure 3, the SAW sensor has a transmitting and receiving transducer which are composed of piezoelectric materials. There is a sensitive membrane between the transmitting and receiving transducers. When an AC signal is input to the transmitting transducer, a two-dimensional acoustic wave with $400 \mathrm{MHz}$ working frequency will be generated and propagated along the crystal surface. Due to the wave interaction, the mass of the membrane will change, which will lead to the fluctuation of wave frequency ultimately. At that point the sensor will record this change and output a similar signal.

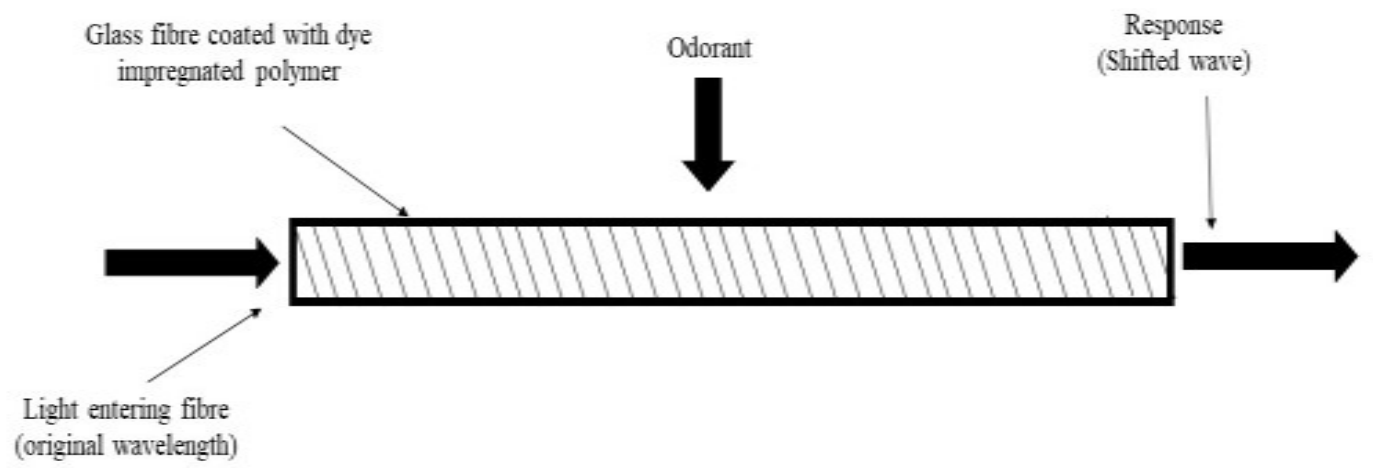

Fig 4. Optic Sensor

Optical sensor is a kind of sensor based on optical principle. It has the characteristics of strong anti-interference capability, non-contact and non-destructive measurement, remote control and high-speed transmission of information [10]. Its working process is relatively simple. When light irradiates volatile organic compounds, a signal will be generated on the sensor. There are two types of optical sensors-intrinsic and extrinsic optical sensors, which could both detect the existence of gaseous compounds and measure their information about scattering, polarization, refractive index and other data. 
SIDE VIEW

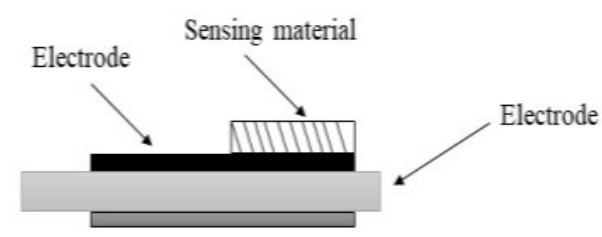

TOP VIEW

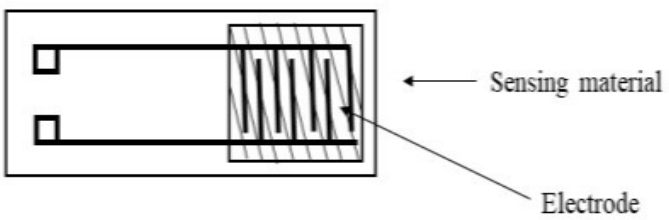

BOTTOM VIEW

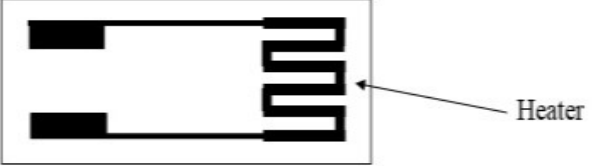

Fig. 5 Gas sensor

As shown in Figure 5, the gas sensor is a kind of converter composed of silicon substrate and conductive polymer coating, which could convert a certain gas volume fraction into corresponding electrical signal [11]. It has the advantages of high sensitivity, easy synthesis, short delay and so on. After the voltage is applied to the sensor, the volatile compounds will change the generated electron flow and in turn cause the change of resistance. This is the exact working principle of the gas sensor.

\subsection{Review on related experiments}

To prove the feasibility of an electronic nose in diagnosing pneumonia, Ümit Özsandikcıoğlu, Şule Yapıcı and Ayten Atasoy carried out a controlled trial in 2018. In order to ensure the accuracy of the result, except for three different subjects, other conditions are exactly the same. Here's a brief introduction of this experiment [12].

In this study, respiratory samples were collected from 46 lung cancer patients, 20 non-smokers healthy people and 10 healthy smokers. In addition, the data normalization method is applied to get the respiratory signals of non-smokers healthy people and healthy smokers respectively. Through the respiratory signal image, people can clearly observe that there are obvious differences, especially in the performance of the sensor TGS2612. Then after series of processing, the feature pair scatter diagram is generated, and lung cancer patients, healthy people and healthy smokers could be distinguished very easily.

This experiment proves the reliability of electronic nose to a certain extent, which means that the diagnosis of lung cancer by electronic nose has entered into a relatively mature stage. The next work is to debug and improve the electronic nose under different working conditions, so as to ensure the stable operation and high precision of the equipment.

\subsection{Review on existing problems}

Although the electronic nose technology has been relatively mature, there are still many problems that cannot be well solved.

First of all, people need to solve the problem of respiratory sampling technology, standard and effective sampling plays a key role in the analysis of exhaled breath. Physiological dead cavity will dilute the concentration of biomarkers, resulting in inaccurate measurement. It seems impractical to breathe in pure air in a period of time before sampling, so people can only try to measure the concentration of VOC in the ambient air and subtract it from the total VOC concentration. Second, the storage of respiratory samples is another big problem. Exhaled breath samples cannot be stored for a long time like histological samples or DNA samples. Currently, the widely used storage methods include Tedlar sampling bags and glass syringes. Using adsorption materials to capture VOC and heat the desorption when necessary is a more promising technology to replace the storage container, which can not only reduce the complexity of the sample, but also realize the sample preconcentration.

Operating temperature is another factor that must be considered when analyzing exhaled gas samples. On the one hand, the working temperature should not be too high to avoid thermal decomposition of some compounds such as aldehydes. This limits some applications of some sensors which need higher working temperature. For this reason, VOC can be extracted and transferred to inert carrier gas environment for measurement or the sensor can be heated locally. On the other hand, at relatively low temperature, macromolecular VOCs are prone to condense, which makes polar VOCs dissolve in water vapor. Therefore, embedding micro hot plate in the 
sensor can ensure the temperature stability in the process of sampling, transportation and measurement.

There are still some shortcomings in the analysis technology of electronic nose system. According to statistics, sampling a single sample in the totality may have negative impacts on the classification results. In the actual measurement, the measurement results of electronic nose system may not come from the biomarkers of lung cancer totally, but from the daily changes. Daily life and exercise (such as food intake, geographical location changes and sleep duration, etc.) can lead to changes in the composition and concentration of exhaled gas, which can greatly affect the accuracy of electronic nose classification [13].

\subsection{Review on prospects}

The clinical analysis of electronic nose in diagnosing lung cancer and other diseases is still in initial stage, but it has attracted more and more attention from the scientific community and clinical medical community. Electronic nose analysis is a non-invasive and rapid test method, which can provide the feedback of disease status and treatment results. Compared with mass spectrometry and other laboratory measurements, electronic nose has great advantages such as exploitability, low measurement cost and small size, which has great potential to significantly reduce medical costs and improve the quality of life. Metal oxide gas sensor technology is mature, and its working temperature, surface modification, structure and morphology optimization can improve the performance of the sensor, which can selectively detect biomarkers related to specific diseases. But at the same time, more efficient communication tools and protocols need to be developed to enable data to be transmitted smoothly between sensor nodes and computing devices, and to provide information support for the precision, integration and intelligence of the whole system [14].

\section{Conclusion}

To identify the presence of chemical components, electronic nose was created. Sensors play a pivotal role in precision in the E-nose system, so that it should be noted that when designing the electronic nose, researchers need to select proper sensors which are not sensitive to temperature, humidity and other environmental conditions, because these conditions are basically not controlled by human beings. Only in this way could people ensure the high accuracy of the electronic nose. In addition, artificial neural network has shown high classification accuracy in higher dimensional problems, which means artificial neural network is the future of E-nose. In the future, the electronic nose can be used to analyse respiratory samples to preliminarily diagnose the risk of lung cancer, and at the same time, using specific mathematical methods and computer processing properly to improve the classification performance is good method.

\section{References}

1. V. H. Tran, H. P. Chan, M. Thurston, P. Jackson, C. Lewis, D. Yates, G. Bell, P. S. Thomas. Breat Analysis of Lung Cancer Patients Using an Electronik Nose Detection System. IEEE Sensors Journal, Vol. 10, No. 9, 2010.

2. E. J. Lewintre, M. Uso, E. Sanmartin, C. Camps. Update on Biomarkers for the Detection of Lung Cancer. Dove Press Journal, 3 (2012), pp. 21-29,2012.

3. Ü. Özsandikcıoğlu, A. Atasoy and Ş. Yapıcı. Hybrid Sensor Based E-Nose For Lung Cancer Diagnosis. In: 2018 IEEE International Symposium on Medical Measurements and Applications (MeMeA), Rome, Italy, 2018, pp. 1-5, doi: 10.1109/MeMeA.2018.8438596

4. G. Peng, U. Tisch, O. Adams, M. Hakim, N. Shehada, Y.Y. Broza, S. Billan, R. Abdah-Bortnyak, A. Kuten, H. Haick. Diagnosing lung cancer in exhaled breath using gold nanoparticles. Nature Nanotechnology, 2009, 4(10), 669-73.

5. Y. Liu, Q. Meng, X. Zhang. Data Processing for Multiple Electronic Noses Using Sensor Response Visualization. In: IEEE Sensors Journal, 2018, vol. 18, no. 22, pp. 9360-9369, 15 Nov.15.

6. R. Hegde and S. Seema. Performance Evaluation and Feature Vector Extraction of Review Data Sets Using Evolutionary Based SVM Techniques. In: 2018 9th International Conference on Computing, Communication and Networking Technologies (ICCCNT), Bangalore, 2018, pp. 1-4.

7. J. Suriya Prakash, K. Annamalai Vignesh, C. Ashok and R. Adithyan. Multi class Support Vector Machines classifier for machine vision application. In: 2012 International Conference on Machine Vision and Image Processing (MVIP), Taipei, 2012, pp. 197-199.

8. F. Reverter, D. Gómez and J. Altet. On-Chip MOSFET Temperature Sensor for Electrical Characterization of RF Circuits. In: IEEE Sensors Journal, 2013, vol. 13, no. 9, pp. 3343-3344.

9. G. C. Frye et al. Optimizing surface acoustic wave sensors for trace chemical detection. In: Proceedings of International Solid State Sensors and Actuators Conference (Transducers '97), Chicago, IL, USA, 1997, vol. 2, pp. 1323-1326.

10. W. Kam, W.S. Mohammed, S. O'Keeffe and E. Lewis. Portable 3-D Printed Plastic Optical Fibre Motion Sensor for Monitoring of Breathing Pattern and Respiratory Rate. In: 2019 IEEE 5th World Forum on Internet of Things (WF-IoT), Limerick, Ireland, 2019, pp. 144-148.

11. Y. Takei, Y. Kanazawa, K. Hirasawa and H. Nanto. Development of 3D gas source localization using multi-copter with gas sensor array. In: 2019 IEEE International Symposium on Olfaction and Electronic Nose (ISOEN), Fukuoka, Japan, 2019, pp. 1-4. 
12. Ü. Özsandikcroğlu, Ş. Yapıcı and A. Atasoy. Classification of Different Breath Samples Utilizing E-Nose System. In: 2018 41st International Conference on Telecommunications and Signal Processing (TSP), Athens, Greece, 2018, pp. 1-5, doi: 10.1109/TSP.2018.8441254.
13. T. Zeng, H. Xu, X. Huang. The progress, challenge and future of exhaled breath sensor. Chinese Journal of Scientific Instrument, 2019, 40 (08): 65-81.

14. L. Zhou, Z.H. Wang, D.C. Sun, et al. Present situation and development of modern precision measurement technology. Chinese Journal of Scientific Instrument. 2017, 38(8): 1869-1878 . 